\title{
Behaviour of Super Conductivity at Various Temperatures
}

\author{
Aditya Sharma $^{1}$, Deepesh Saxena ${ }^{2}$ \\ B.E. Students, Electronics and Communication, LNCTS (RIT), Indore, India ${ }^{1,2}$
}

\begin{abstract}
The high temperature superconductivity has a massive potential for technological use since its discovery. But the mechanism of high temperature superconductivity still remains hard to define; a lot of development has been made resulting in a major reduction in the number of proposed mechanisms under consideration. At present, electron phonon interaction or spin fluctuations are considered to be principal of the mechanism of high temperature superconductivity. While the transition temperature has not been improved over the last few years and we are unable to see room temperature superconductivity in near future, but many of the technological applications are still in use and being constantly improved. New materials with a better possibilities are also being discovered. This paper attempts to explain the progress made till know and shed light on the challenges of understanding the working of superconductors and achieving room temperature superconductivity.
\end{abstract}

Keywords: Superconductivity, HTSC (High Temperature Superconductivity), Critical Temperature $\left(\mathrm{T}_{\mathrm{C}}\right)$, Cuprates.

\section{INTRODUCTION}

In 1908, Heike Kamerlingh Onnes in Holland found the way toliquefy helium and to reach temperatures as low as $4 \mathrm{~K}$. In 1911, he discovered that below a temperature of the order of $4.2 \mathrm{~K}, \mathrm{Hg}$ (mercury) looses the resistivity[1]. His discoveries lead him to realize that he was in the presence of a new state of solid mater. He could establish that when a particular magnetic field than rely on temperature, the critical field, $\mathrm{H}_{\mathrm{c}}(\mathrm{T})$, was applied, the standard properties of the metal were recovered. Also a critical current, $\mathrm{J}_{\mathrm{c}}(\mathrm{T})$, could have the same effect. He called that new phenomena, superconductivity.

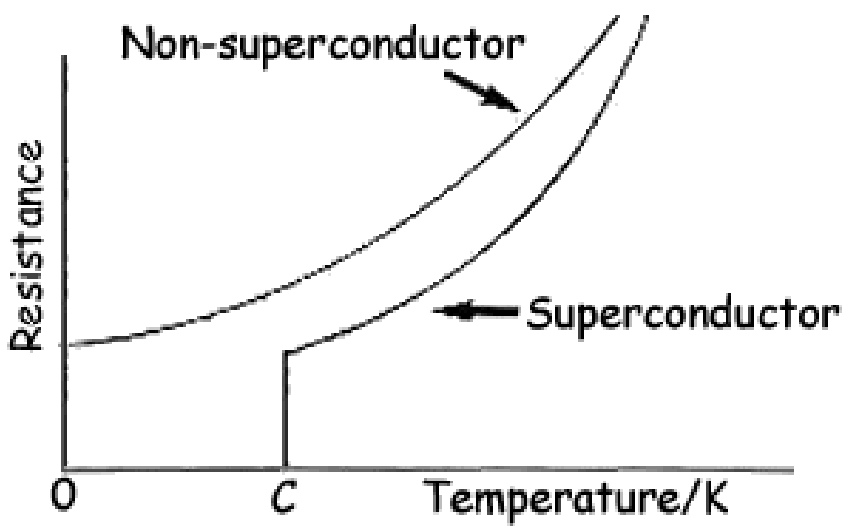

$C=$ critical temperature

Fig. 1. Superconductivity Characteristics

\section{II.PREPROCESSING}

The new phase has many new materials have been found which shows this phenomenon. Today over a thousand materials, including several pure chemical elements, are discovered to become superconductors at various temperatures ranging up to about $20 \mathrm{~K}$, but not at room temperature.

Within the last several years a new family of ceramic compounds has been revealed which are insulators at room temperature and superconductors at temperatures of liquid nitrogen. Which shows that far from being a exceptional physical phenomenon, superconductivity is a fairly common property of most of the materials. 
Vol. 5, Issue 4, April 2017

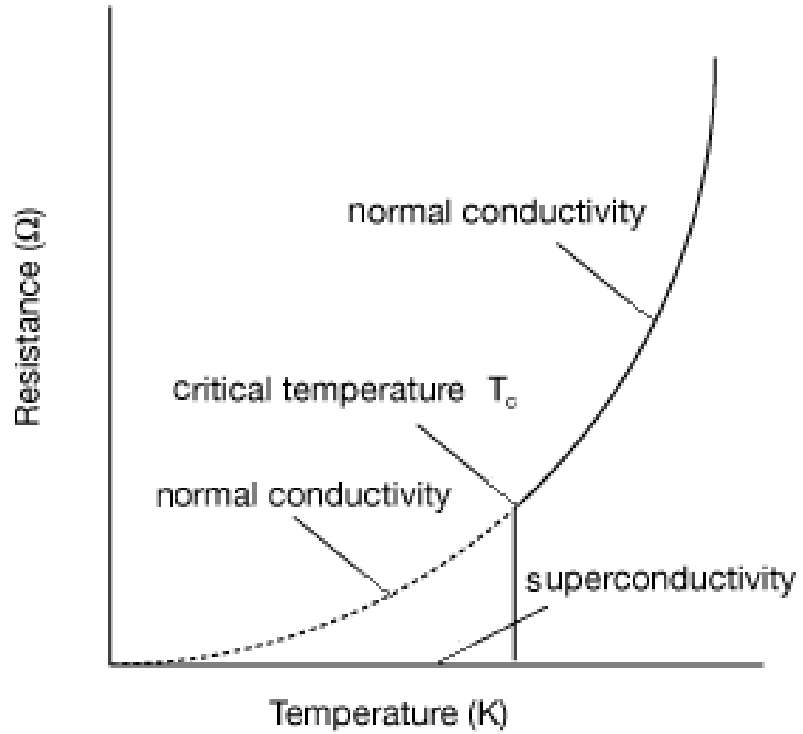

Fig. 2. Superconductivity Properties

Heike Kamerlingh Onnes description was successful when, in 1913, he concluded superconductivity in lead and tin (also at $4 \mathrm{~K}$ ). This excited Onnes very much because now he had established that other substances had the property to form the superconductors. Not only that, these substances were easily drawn into wires example $\mathrm{Hg}$ (mercury). The potential to produce superconducting devices was immediately apparent to Onnes. 'Tin and lead being easily workable materials, we can now anticipate all kinds of electrical experiments with apparatus without resistance.'Onnes, 1913 Onnes received the Nobel prize in 1913 for discovery of phenomena of super conductivity.

\section{A. THE MEISSNER EFFECT}

The Meissner effect, Walter Meissner and Robert Ochsenfeld in 1933 discovered a magnetic phenomenon that showed that superconductors are not just perfect conductors. The thought experiment that highlights this difference as Type I and Type II Superconductors. Imagine that both the ideal conductor under the ideal condition and superconductor are above their critical temperature, Tc. That is, they both are in a normal conducting state and have electrical resistance. A magnetic field, $\mathrm{Ba}$, is then applied in order to observe this phenomena[2]. This results in the field penetrating both materials. Both samples are then cooled so that the ideal conductor now has zero resistance. It is noted that the superconductor expels the magnetic field from inside it, while the ideal conductor maintains its interior field.

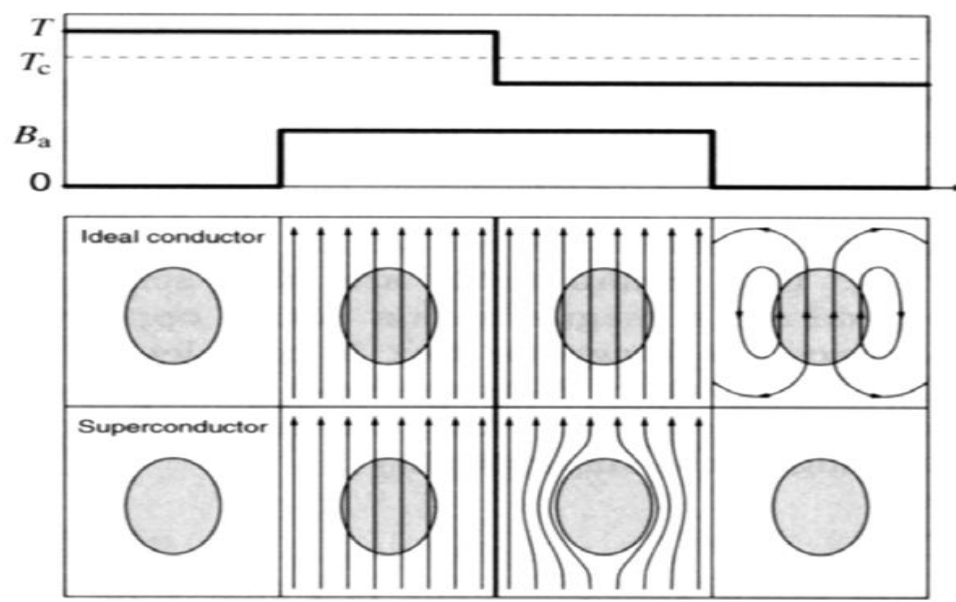

Fig. 3. The Meissner Effect

Note that energy is needed by the superconductor to debar the magnetic field. This energy comes from the exothermic superconducting transition. Switching off the field induces currents in the ideal conductor that prevent changes in the magnetic field inside it according to Lenz's law. However, the superconductor returns to its initial state, i.e. no magnetic field inside or outside it[4]. 


\section{ISO 3297:2007 Certified}

Vol. 5, Issue 4, April 2017

\section{MECHANISM}

The properties exhibited by conventional superconductors were very well explained by the BCS theory .This theory envisages an attractive interaction between electrons mediated by phonons resulting in the formation of the so called Cooper pairs. The electrons in these pair states are no longer required to obey the Fermi-Dirac statistics. The theory was very successful, making many predictions that were quicklyconformed by experiment. For most of the conventional superconductors this theory satisfactorily explains the properties like Isotope Effect, Specific heat Jump, Penetration Depth, the Energy Gap, Coherence Length and others as summarized in[4].

The BCS theory was further validated by the flux quantization measurement and Josephson effect both of which suggested that super-currents involve pair of electrons. However, the theory also implied that the forces binding the Cooper pairs were very feeble, so they would be tear apart by thermal vibrations at anything other than extremely low temperatures and therefore superconductivity might not occur above $30 \mathrm{~K}$.

\section{A. BCS THEORY AND COOPER PAIRS}

Theories in modern physics that can explain experimental phenomena across 10 or more orders of magnitude in energy scales are very few. The Bardeen, Cooper, and Schrieffer (BCS) theory of superconductivity is such a theory that works over a massive range of systems, from a very small scale (nuclei) up to a very large scale (neutron stars), with all the systems between them[1]. The structure developed by John Bardeen, Leon Cooper, and J. Robert Schrieffer was, after all, intended to answer a apparently unimaginative question: Why is it that if we immerse a piece of common metal, such as $\mathrm{Pb}, \mathrm{Al}$, or $\mathrm{Hg}$, in liquid $\mathrm{He}$, we cannot measure any trace of resistance? The answer to this apparently benign question eluded Albert Einstein, Felix Bloch, Werner Heisenberg, and others of their calibre for more than four decades. The BCS landmark paper, which was published in 1957, sheds light on these previous unsuccessful attempts. The methods of modern many-body theory, necessary for a proper treatment of the interaction between electrons and the lattice, had not been developed until then.

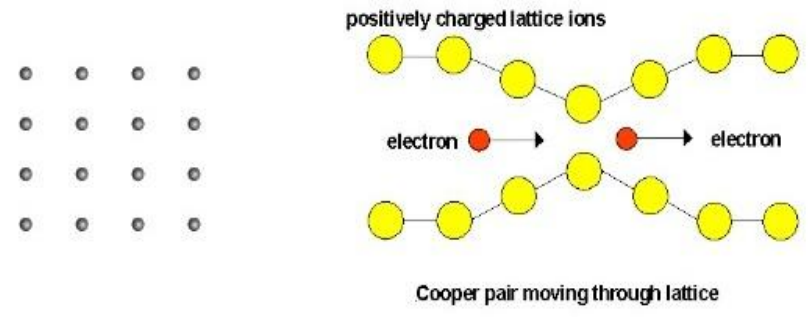

Fig. 3. The Meissner Effect

It turns out that this macroscopic coherence of microscopic quantum objects (the electrons) is possible only if the electrons are glued into pairs and if the pairs mutually overlap. The bottom figure illustrates the idea of electronic pairing by lattice vibrations. Ions in a metal are positively charged, and the mobile electrons have a negative charge; therefore, ions around an individual electron are attracted to it, creating a more concentrated positive charge around the electron[3].

Because an electron moves so much faster than an ion, by the time the slow ions complete their travel toward the electron, the electron has already left this area, leaving behind the cloud of displaced ions with their net positive charge. Of course, when the ions realize that they were being attracted to an object that is no longer there, they start moving back to their original locations. However, this takes time. Within this time, another electron can be attracted to this cloud of positive charge[5].

If the time scales are properly tuned, the second electron can trace the path of the first one, following it around the crystal. In such a situation, we say that the electrons have formed a "Cooper pair."

\section{III.LITERATURE SURVEY}

A. Superconductivity above $130 \mathrm{~K}$ in the HG-BA-CA-Gu-O system Schilling, M. Gantoni, J. D. Guo \& H. R. Отт

The author demonstrated about superconductivity below a transition temperature of $94 \mathrm{~K}$ in $\mathrm{HgBa} 2 \mathrm{CuOa} * 6$ has enlarged the repertoire of high-7" superconductors consisting copper oxide planes merged in suitably layered materials. 


\section{ISO 3297:2007 Certified}

Vol. 5, Issue 4, April 2017

Previous experience with identical compounds containing bismuth andthallium instead of mercury recommended that even higher transition temperatures might be accomplished in mercury-based compounds with more than one $\mathrm{CuO}$, layer per unit cell. Thus we conclude that at present we cannot relate the different superconducting stage to crystallographic phases. There is no uncertainty proof that the occurrence of superconductivity in our samples stems from the $\mathrm{HgBa} 2 \mathrm{Ca},-1 \mathrm{Cu}, \mathrm{O} 2$, ar*u phases.

\section{B. High Temperature Superconductivity: Materials, Mechanism and Applications- M.A. Malik, B.A. MALIK}

The author states that superconductivity is a phenomenon contest by certain materials by virtue of which they drop all their electric resistance beneath certain sufficiently low temperature attend by a total expulsion of magnetic field from within. Thus we conclude that the technological uses of high temprature superconductivity are immense, a main benefit is that because of their ability to reduce size and weight and in the method save energy, high temprature superconductivity promises to show the global environmental issues as well.

\section{IV.PROPOSED METHOD}

\section{A. High Temperature Superconductivity - M. Brian Maple}

The author demonstrated about the discovery of superconductivity at $\sim 30 \mathrm{~K}$ in the La-Ba-Cu-O system ignited an explosion of interest in high temperature superconductivity. These initial developments rapidly evolved into an intense worldwide research effort that still persists after more than a decade, fueled by the fact that high temperature superconductivity constitutes an extremely important and challenging intellectual problem and has enormous potential for technological applications. Thus we conclude that during the past decade, remarkable progress in the areas of basic research and technological applications has been made on the high Tc cuprate superconductors. The availability of high quality poly-crystalline and single crystal bulk and thin film materials has made it possible to make reliable measurements of the physical properties of these materialsand to optimize superconducting properties (e.g., $\mathbf{J}_{\mathrm{c}}$ ) that are important for technological applications.

\section{B. A BREAKTHROUGH OF THE CENTURY}

Later on, four individuals of the Tanaka group, who were later called the "Gang of Four"-Shoji Tanaka, Koichi Kitazawa, Shinichi Uchida, andHidenori Takagi, depending up on previous diagram scientists worked twenty four hours on intense research that resulted first of all in the confirmation of Meissner effect, and identification of the chemical composition and crystal structures that ready the platform for the phenomenon of superconductivity to appear. In early December that year.

Kitazawa presented these findings at the MRS Meeting in Boston, effectively sparking a firestorm of interest worldwide[3].

This was a "breakthrough of the century" that prompted the fast-track launch, in FY1987, of another project in Specific Research, on the "Sankabutsu koon chodendotai no kenkyu" (writtten report on high-temperature superconducting oxides), following in the footsteps of the earlier Specific Research project on "Shin chodendo busshitsu" (new superconducting materials)

The "Pack of Four" responsible for tend the fever of curiosity in copper-oxide superconductivity. : Kitazawa,Tanaka, Uchida, and Takagi. (1986)with the breakthrough of novel copper-oxide compounds, the critical temperature at which superconductivity sets in bush initially from 23 Kelvin to 30 Kelvin, and then, in the spring of 1987,leaped all the way to 100 Kelvin. Because this was more than the $-196 \mathrm{C}$ boiling point of liquid nitrogen, the prospects for commercial applications ofsuperconductivity attracted broad public interest and spurred a widening global race to find materials with even higher critical temp. The high- temperature superconductivity "fever" had taken hold[2].

\section{APPLICATIONS}

The practical applications of conventional superconductors are limited due to the very low operating temperature. The discovery of higher Tc materials extends the feasible applications of superconductors. These applications include highspeed trains, magnetic energy storage, magnetic resonance imaging (MRI) for medical applications, Josephson devices, Superconducting Quantum Interference Devices (SQUID), Magneto encephalography, microwave devices and resonators to high energy physics experiments. SQUIDs deserve a special mention because of their versatility finding applications in magnetic materials characterization, medicine, NMR, MRI, geophysics, quantum computing etc. SQUID magnetometers may be the most sensitive measurement device known. 


\section{ISO 3297:2007 Certified}

Vol. 5, Issue 4, April 2017

The threshold for SQUID is of the order of $1 \mathrm{fT}$, making it capable of measuring extremely feeble magnetic fields. In comparison, magnetic field of heart is around 50,000 fT and that of brain is a few fT. Because of their extreme sensitivity, SQUIDs have established themselves as very accurate devices for both Magneto cardiography and Magneto encephalography. SQUIDs are being considered for a search for the Dark Matter. The idea is that when axioms of a given mass/energy enter a microwave cavity sited from a liquid helium-cooled superconducting solenoid, they will interact with the field and decay into photons. These photons can then be amplified and detected using extremely sensitive SQUIDs. Such experiments are underway in the university of Washington.

The other prospective application of superconductivity is lossless Power Transmission. In India, 22\% of electric power is lost in transmission alone. A superconducting wire can transmit a dc current without losses or an ac current with an extremely small loss. In the west, people are contemplating to utilize the benefit of superconductivity in transporting energy on a scale of many gigawatts to terawatts from remote generation facilities (the Sahara desert), which otherwise would be futile owing to huge transmission losses[3]. The fact that HTSC are extreme type II, characterized by very high upper critical field values, opens up the possibility of superconducting Maglev Trains.

Superconducting Motors and generators could be made with a weight of about one tenth that of conventional devices for the same output. The basic elements of the world's first "quantum" computer are superconducting quantum bits. In the Fermilab, superconducting magnets were successfully used to increase the ultimate particle energy and the operating cost was greatly reduced. Recently, experts from the CERN Superconductors team obtained a world record current of $20 \mathrm{kA}$ at $24 \mathrm{~K}$ in an electrical transmission line consisting of two 20-metre long cables made of $\mathrm{MgB} 2$ superconductor. This result makes the technology a viable solution for long-distance power transportation.

\section{VI.CONCLUSION}

Since the discovery of superconductivity in the cuprate family La-Ba-Cu-O ( $\mathrm{T}_{\mathrm{c}}$ up-to $35 \mathrm{~K}$ ), hundreds of cuprate oxide superconductors have been synthesized with varying advantages. An exhaustive description of almost all High Tc superconducting cuprate families. We shall here mention only some of these cuprate families in order of increasing transition temperatures. Soon after the breakthrough of Bednorz and Muller, Wu et al discovered super conductivity in $\mathrm{Y}-\mathrm{Ba}-\mathrm{Cu}-\mathrm{O}$ system with transition temperature well above the liquid nitrogen temperature $(\mathrm{Tc}=93 \mathrm{~K}$ in $\mathrm{YBa} 2 \mathrm{Cu} 3 \mathrm{O} 7-\mathrm{x}, \mathrm{x}=0.07)$. Later, super conductivity was discovered in the $\mathrm{Bi}-\mathrm{Sr}-\mathrm{Ca}-\mathrm{Cu}-\mathrm{O}$ system at a transition temperature of above $105 \mathrm{~K}$.In the same year, Thalium based cuprates were found to show superconductivity at $120 \mathrm{~K}$ . Further, it followed that TC could be enhanced by increasing the number of conducting $\mathrm{CuO} 2$ layers. Another class of the Carbon based families-the oxy carbonates could show superconductivity up to a $\mathrm{Tc}$ of $117 \mathrm{~K}$. The critical temperature reached a value of $133 \mathrm{~K}$ in Mercury based cuprates[4]. The transition temperature could further be increased by applying high pressure. At present the record high transition temperature is $164 \mathrm{~K}$ in Mercury based cuprates under high pressure which is within a factor of two of room temperature.

A team of researchers at MIT have reported the existence of charge density waves that would explain the phenomenon of high Tc superconductivity and hence lead to room temperature superconductivity. Kawashima suggest that room temperature superconductor may be obtained by bringing alkanes into contact with a graphite surface. Room temperature superconductivity in substitutionally doped graphene via a combined mechanism involving phononic and electronic processes has been reported by Sinha and Jindal . However, as of now, there is no conformation of a reproducible room temperature superconductivity and the only materials with $\mathrm{Tc}$ above the liquid Nitrogen temperature are still the copper oxide superconductors.

\section{ACKNOWLEDGMENT}

We would like to acknowledge the following research papers and related topics regarding Superconductivity as mentioned in the following references which are given below:

\section{REFERENCES}

[1] Superconductivity by Joe Khachan and Stephen Bosi.

[2] Theory of Superconductivity Carsten Timm

[3] H M Rosenberg - The Solid State. http://www.amazon.co.uk/Solid-State-Introduction-MaterialsEngineering/dp/0198518706/ref=sr_1_1/20202107608966217?ie=UTF8\&s=books\&qid=1183800926\&sr=1-1 This is gives a very basic introduction to the phenomena of superconductivity.

[4] Campbell, A.M. and Cardwell, D.A. and Ashworth, S.P. and Coombs, T.A. (1994)," Power engineering applications of high temperature superconductors," IRC in Superconductivity Research Review 1994 


\section{IJIREEICE \\ Vol. 5, Issue 4, April 2017 \\ [5] High Temperature Superconductivity: Materials, Mechanism and Applications- M.A. Malik, B.A. Malik \\ [7] Superconductivity above $130 \mathrm{~K}$ in the Hg-Ba-Ca-Gu-O system A. Schilling, M. Gantoni, J. D. Guo \& H. R. Ott}

[6] High Temperature Superconductivity - M. Brian Maple

\section{BIOGRAPHIES}

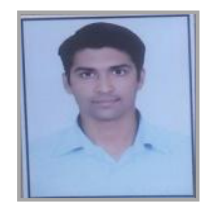

I am Aditya Sharma, student of Bachelor's of Engineering in Electronics and Communication department. Current I am in the final year of my course assessment from LNCTS (RIT) Indore. I prefer Superconductivity because this has a huge technological potential in modern era.

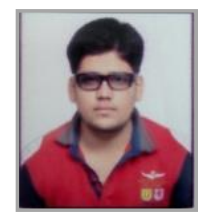

I am Deepesh Saxena, student of Bachelor's of Engineering in Electronics and Communication department. Current I am in the final year of my course assessment from LNCTS (RIT) Indore. I decided to write a research paper to get better opportunities in higher studies. 